\title{
Abordagem mista em teses de um programa de pós-graduação em educação: análise à luz de Creswell ${ }^{1}$
}

\author{
Luciana Rodrigues Leite ${ }^{2}$ \\ ORCID: 0000-0003-1915-6462 \\ Ana Paula dos Santos Reinaldo Verde ${ }^{3}$ \\ ORCID: 0000-0002-8138-7280 \\ Francisco das Chagas Rodrigues de Oliveira ${ }^{4}$ \\ ORCID: 0000-0002-3478-1058 \\ João Batista Carvalho Nunes ${ }^{5}$ \\ ORCID: 0000-0002-1270-0026
}

\section{Resumo}

A pesquisa de métodos mistos engloba a associação entre procedimentos de coleta, análise e combinação de técnicas quantitativas e qualitativas. Nos últimos anos ocorreu um aumento vertiginoso no uso da abordagem mista, sobretudo na literatura internacional. Este ensaio incide sobre o uso do método misto na pesquisa em educação, no Brasil, com fundamento na seguinte questão norteadora: "em que medida a abordagem mista de pesquisa está inserida, no Brasil, em programas de pós-graduação da área de educação (PPGEs), considerados de desempenho equivalente a padrões internacionais de excelência?". Desenvolveu-se, portanto, pesquisa documental, na qual foram mapeadas e analisadas teses de um programa de pós-graduação, nota sete, de acordo com avaliação da Coordenação de Aperfeiçoamento de Pessoal de Nível Superior (CAPES), no período de 2013 a 2018. Os estudos de John Creswell forneceram a base teórica e os critérios de análise das teses selecionadas. As buscas foram realizadas na Plataforma Sucupira e, dentre as 104 teses identificadas como pertencentes ao PPGE em foco, no referido período, $11(10,6 \%)$ foram identificadas como concernentes ao contexto das investigações do tipo mistas. Considera-se que há, em maior e menor grau, a percepção do uso potencial de um projeto de método misto nas teses analisadas, com evolução temporal na melhoria dessa percepção, pois teses mais recentes apontam justificativas mais claras para a integração quali-quantitativa. Identificou-se também evolução teórica e metodológica; todavia, há

\footnotetext{
1- Disponibilidade de dados: todo o conjunto de dados que dá suporte aos resultados deste estudo foi publicado no próprio artigo.

2- Universidade Estadual Vale do Acaraú, Sobral, Ceará, Brasil. Contato: luciana_leite@uvanet.br.

3- Secretaria de Educação do Estado do Maranhão, São Luís, Maranhão. Contato: napaulareinaldo@gmail.com.

4- Rede Municipal de Boa Viagem, Ceará e Rede Municipal de Monsenhor Tabosa, Ceará, Brasil. Contato: chicoverde.ufjj@hotmail.com.

5- Universidade Estadual do Ceará, Fortaleza, Ceará, Brasil. Contato: joao.nunes@uece.br.
} 
necessidade de os pesquisadores do referido programa buscarem referências mais atuais no tocante aos estudos do tipo misto.

\section{Palavras-chave}

Abordagem mista - Pesquisa em educação - Pós-graduação em educação - Teses.

\section{Mixed methods approach in the theses of a postgraduate program in education: analysis in the light of Creswell*}

\section{Abstract}

Mixed methods research embraces the association of procedures to collect and analyze data as well as the combination of quantitative and qualitative techniques. In the past years there has been an increase in the use of mixed methods, especially in the international literature. This article focuses on the use of mixed methods to education research in Brazil. The following question guides the investigation: "to what extent the mixed methods approach is present in Brazilian Postgraduate Programs in Education whose academic performance matches the international standards of excellence?". A documentary research was conducted to map and analyze the theses of a postgraduate program that reached the highest score according to Coordination for the Improvement of Higher Education Personnel (CAPES) from 2013 to 2018. The studies of John Creswell provided our theoretical basis and the analysis criteria for the selected theses. The search conducted on the Sucupira Platform found that 11 of 104 theses (10.6\%) from the appointed Postgraduate Program in Education make use of mixed methods. Thus, it was verified, to greater and lesser degrees, the perception of the potential use of mixed methods in the theses, which improves over time as more recent theses present clearer justifications for integrating qualitative and quantitative techniques. In addition, both theoretical and methodological developments were verified, however, researchers in the Postgraduate Program have yet to employ more up to date references regarding mixed methods research.

\section{Keywords}

Mixed methods - Education research - Post-graduation in education - Theses. 


\section{Introdução}

Este artigo mapeia e analisa a aplicação da abordagem mista de pesquisa em teses de um programa de pós-graduação em educação brasileiro, nota sete, de acordo com avaliação da Coordenação de Aperfeiçoamento de Pessoal de Nível Superior (CAPES). Para a elaboração deste escrito, serviram como arrimo os pressupostos de Creswell (2012), para quem a pesquisa de métodos mistos engloba a associação entre procedimentos de coleta, análise e combinação de técnicas quantitativas e qualitativas que, mediante seu caráter interdisciplinar, são um passo adiante, pois a utilização dos pontos fortes de cada uma dessas abordagens encaminha-se recorrentemente para a elaboração de investigações de qualidade superior. As perspectivas desse autor também fundamentaram todo o percurso de análise dos textos selecionados, visto que foi com suporte em suas orientações - acerca das características que orientam a elaboração e validação de um estudo do tipo misto que esta pesquisa foi edificada.

John W. Creswell é professor de Psicologia Educacional no Programa de Pósgraduação em Métodos Quantitativos e Qualitativos em Educação (MQQE) da University of Nebraska-Lincoln. Justifica-se a escolha deste teórico para fundamentar a tessitura deste artigo mediante seu reconhecido trabalho na seara de metodologia da pesquisa, haja vista que ele é autor de inúmeras publicações, inclusive livros traduzidos para outros idiomas aos quais se recorre em todo o mundo; e, sobretudo, por ser referência em pesquisa de métodos mistos, atuando intensivamente na difusão deste enfoque de busca ordenada do conhecimento.

0 gérmen para a elaboração desta proposta investigativa emergiu da compreensão de que o emprego da abordagem mista, máxime em artigos, cresceu vertiginosamente nos últimos tempos. Galvão, Pluye e Ricarte (2018) destacam que essa realidade é ainda mais acentuada no panorama internacional, o que é um indicador do caminho a ser percorrido por investigadores brasileiros e latino-americanos. Nesse contexto, este ensaio foi edificado com fundamento na seguinte questão norteadora: em que medida a abordagem mista de pesquisa está inserida, no Brasil, em programas de pós-graduação da área de Educação, considerados de desempenho equivalente a padrões internacionais de excelência?

À demanda de respostas para o expresso questionamento, a investigação sob relato foi norteada por indagações específicas, dispostas a seguir: a) qual a incidência de utilização do método misto em teses de um programa de pós-graduação em educação (PPGE), nota sete, na avaliação da CAPES?; b) quais pressupostos fundamentaram o desenho metodológico desses estudos?; c) em que medida essas teses se harmonizam às orientações metodológicas de Creswell, para a organização de uma pesquisa de métodos mistos?

Delimitou-se a pesquisa a um programa de pós-graduação nota sete, na avaliação da CAPES, por este ser um indicativo do alto padrão de desempenho esperado das teses defendidas nesse ambiente investigativo. Fato que ressalta a importância de se inventariar e analisar essas produções, no intuito de favorecer o fortalecimento da pós-graduação em educação no Brasil, e, sobretudo, agregar contributos ao rigor das produções na área.

Com efeito, no intuito de resguardar o rigor científico e metodológico deste estudo, inicialmente foi delimitado o recorte temporal de 2013 a 2018. As buscas foram realizadas 
no âmbito da Plataforma Sucupira e, dentre as 104 teses identificadas como pertencentes ao PPGE em foco, no referido período, $11(10,6 \%)$ foram identificadas como concernentes ao contexto das investigações do tipo mistas. Estas, a seu turno, foram analisadas à luz dos pressupostos teóricos de John Creswell, mediante critérios expostos na seção metodológica deste escrito.

Por conseguinte, a mostra do relatório deste estudo foi organizada em quatro seções. Na primeira, são indicados os pressupostos teóricos que fundamentaram esta investigação, traçando um breve histórico da ascendência de buscas sob métodos mistos, suas definições, características e desafios que se impõem ao investigador ao operar esse enfoque. No módulo seguinte, cuida-se acerca dos aspectos metodológicos adotados no percurso da pesquisa, para, na sequência, realizar a exposição e análise das teses que recorrem à abordagem mista. No segmento de fechamento - o quarto - apontam-se as contribuições deste experimento para o terreno do conhecimento no qual está inserido e as principais conclusões a que se chegou mediante a análise dos textos acadêmicos stricto sensu selecionados.

\section{Pressupostos teóricos acerca da pesquisa de métodos mistos}

Uma visão atenta sobre as publicações em periódicos nacionais e internacionais das mais diversas áreas do saber parcialmente ordenado demonstra que estudos envolvendo o entrelaçamento de métodos qualitativos e quantitativos são cada vez mais comuns. 0 ascendente próprio à pesquisa de métodos mistos é uma realidade ressaltada por autores como Creswell (2013) e Ivankova (2015), ao apontarem o aumento vertiginoso da popularidade desse estalão de estudo.

Nessa perspectiva, afigura-se relevante mostrar algumas características relacionadas à evolução do conceito de pesquisa de métodos mistos. Creswell e Clark (2013) oferecem panorama interessante acerca desta realidade, e destacam que, de acordo com o aumento do interesse dos pesquisadores por esse método, emergiram, paulatinamente, várias definições que, por sua vez, incorporam elementos dos métodos em si (qualitativos e quantitativos), dos procedimentos de busca, da filosofia e do projeto de pesquisa.

Inicialmente, a pesquisa de métodos mistos de Creswell e Clark (2013) foi caracterizada como a mistura de dois procedimentos metodológicos, sendo autores do terreno da avaliação que, em 1989, mostraram esta definição - a primeira - sem relacioná-la às concepções filosóficas que fundamentam esse enfoque. Somente dez anos depois, Tashakkori e Teddlie (1998) denotam uma definição mais ampla, caracterizando-a como orientação metodológica em que os dois métodos (qualitativo e quantitativo) são misturados em todas as fases da pesquisa, desde a coleta, análise dos resultados, até posições filosóficas.

Com o passar do tempo e a popularização de investigações sob métodos mistos, uma diversidade de definições foi pouco a pouco disseminada. Algumas delas resultam mais relacionadas a orientações de como desenvolver estudos do tipo misto. Exemplo 
desse modelo de definição está em Tashakkori e Creswell (2007, p. 4), que a compreendem "[...] como pesquisa em que o investigador coleta e analisa os dados, integra os achados e extrai inferências usando abordagens ou métodos qualitativos e quantitativos em um único estudo ou programa de investigação" .

Refletindo, então, nesta multiplicidade de perspectivas a serem consideradas, Creswell e Clark (2013) formularam uma definição, cobrindo as características essenciais da pesquisa de métodos mistos. Esta, por sua vez, combina métodos, uma filosofia e uma orientação do projeto, além de destacar os componentes fundamentais que integram o planejamento e a condução de um estudo de métodos mistos. Segundo os autores,

[...] o pesquisador coleta e analisa de modo persuasivo e rigoroso tanto os dados qualitativos quanto os quantitativos (tendo por base as questões de pesquisa); mistura (ou integra ou vincula) as duas formas de dados concomitantemente, combinando-os (ou misturando-os) de modo sequencial, fazendo um construir o outro ou incorporando um no outro; dá prioridade a uma ou a ambas as formas de dados (em termos do que a pesquisa enfatiza); usa esses procedimentos em um único estudo ou em múltiplas fases de um programa de estudo; estrutura esses procedimentos de acordo com visões de mundo filosóficas e lentes teóricas; e combina os procedimentos em projetos de pesquisa específicos que direcionam o plano para a condução do estudo. (CRESWELL; CLARK, 2013, p. 22).

Para confirmar a perspectiva desses autores, Sampieri, Collado e Lúcio (2013, p. 557) ressaltam que "um estudo misto sólido começa com a formulação de um problema contundente e demanda claramente o uso e integração do enfoque quantitativo e do qualitativo [...]”. Estes, por sua vez, se integram desde a formulação do problema, passando pela amostragem, pela coleta de dados, pelos procedimentos de análise desses dados e pela interpretação dos resultados. Com efeito, Creswell (2010) aponta quatro aspectos importantes a serem considerados pelo pesquisador no planejamento de um estudo à maneira do agora relatado: distribuição do tempo, atribuição de peso, combinação e teorização, conforme explicitado no Quadro 1. Para facilitar a descrição dessas estratégias, foi desenvolvida uma notação dos métodos mistos, representada por meio de rótulos e símbolos abreviados, utilizados pelos pesquisadores para comunicar seus procedimentos. Creswell e Clark (2013) indicam a utilização de $(+)$ para estudos concomitantes e $(\rightarrow)$ para estudos sequenciais; 'Qual' e 'Quan' representam qualitativo e quantitativo, respectivamente, e são identificados com letras maiúsculas ou minúsculas para indicar o peso ou prioridade de cada um desses métodos no estudo. Assim, a notação QUAN/qual, por exemplo, significa que o método quantitativo tem maior peso nesse estudo.

\footnotetext{
6- "[...] as research in which the investigator collects and analyzes data, integrates the findings, and draws inferences using both qualitative and quantitative approaches or methods in a single study or a program of inquiry".
} 
Quadro 1 - Aspectos a serem considerados no planejamento de um estudo misto

\begin{tabular}{|c|c|c|c|}
\hline DISTRIBUIÇÃO DO TEMPO & ATRIBUIÇÃO DE PESO & COMBINAÇÃO & TEORIZAÇÃO \\
\hline $\begin{array}{c}\text { A coleta dos dados } \\
\text { qualitativos e quantitativos } \\
\text { ocorrerá ao mesmo tempo } \\
\text { (concomitante) ou em fases } \\
\text { (sequencial) }\end{array}$ & $\begin{array}{l}\text { Refere-se ao peso ou prioridade } \\
\text { atribuídos aos âmbitos } \\
\text { qualitativo e quantitativo. O peso } \\
\text { pode ser igual ou enfatizar um } \\
\text { ou outro. } \\
\text { (QUAL-QUAN; } \\
\text { QUAL-quan; } \\
\text { QUAN-qual) }\end{array}$ & $\begin{array}{l}\text { Refere-se aos estágios em que a combinação } \\
\text { de dados qualitativos e quantitativos ocorrerá: } \\
\text { coleta, análise, interpretação ou nas três fases. } \\
\text { Conectados - dados quali e quanti são } \\
\text { conectados durante as fases da pesquisa } \\
\text { Integrados - dados quali e quanti são } \\
\text { fundidos e se apoiam mutuamente } \\
\text { Incorporados - dados secundários apoiam } \\
\text { um banco de dados principal. }\end{array}$ & $\begin{array}{l}\text { Se o estudo é guiado } \\
\text { ou não por uma } \\
\text { perspectiva teórica } \\
\text { com maior alcance, } \\
\text { podendo ser explícita } \\
\text { ou implícita. }\end{array}$ \\
\hline
\end{tabular}

Fonte: Adaptada de Creswell (2010).

Para Creswell (2010), esses quatro fatores ajudam na edificação da estratégia de métodos mistos adotada na investigação. Uma estratégia de pesquisa, segundo Yin (2005), define o modo como os dados serão coletados e analisados. No estudo específico ora examinado, Creswell et al. (2003) apontam a existência de seis estratégias principais para os pesquisadores escolherem ao planejarem uma proposição de busca ordenada (embora não se esgotem todas as possibilidades). Os estudos sequenciais se dividem em: estratégia explanatória sequencial, estratégia exploratória sequencial e estratégia transformativa sequencial; enquanto aqueles que utilizam uma perspectiva concomitante dividem-se em estratégia de triangulação concomitante; estratégia incorporada concomitante e estratégia transformativa concomitante, conforme características dispostas no Quadro 2.

Quadro 2 - Estratégias de pesquisa de métodos mistos

\begin{tabular}{|c|c|c|c|c|}
\hline $\begin{array}{c}\text { ESTRATÉGIA } \\
\text { sequencial }\end{array}$ & PESO & COMBINAÇÃO & TEORIZAÇÃO & DEFINIÇÃO \\
\hline $\begin{array}{c}\text { Exploratória } \\
\text { Sequencial }\end{array}$ & Qualitativo & Conectados & Implícita & $\begin{array}{c}\text { A coleta e análise de dados quantitativos ocorre inicialmente, } \\
\text { seguida da coleta e análise de dados qualitativos. Essa segunda } \\
\text { fase é desenvolvida com base nos resultados quantitativos } \\
\text { iniciais. }\end{array}$ \\
\hline $\begin{array}{c}\text { Transformativa } \\
\text { Sequencial }\end{array}$ & $\begin{array}{c}\text { Qualitativo ou } \\
\text { Quantitativo } \\
\text { seguida da coleta e análise de dados quantitativos. Essa segunda }\end{array}$ & Conectados & Explícita & $\begin{array}{c}\text { Pode iniciar pela parte qualitativa ou quantitativa e diferencia- } \\
\text { se das demais estratégias por possuir uma perspectiva teórica } \\
\text { norteadora do estudo. }\end{array}$ \\
\hline $\begin{array}{c}\text { Triangulação } \\
\text { concomitante }\end{array}$ & $\begin{array}{c}\text { Qualitativo e } \\
\text { Quantitativo } \\
\text { (igual) }\end{array}$ & Integrados & $\begin{array}{c}\text { Explícita ou } \\
\text { implícita }\end{array}$ & $\begin{array}{c}\text { Coleta de dados qualitativos e quantitativos de modo } \\
\text { concomitante (ao mesmo tempo) e posterior comparação a } \\
\text { bancos de dados. }\end{array}$ \\
\hline $\begin{array}{c}\text { Incorporada } \\
\text { concomitante }\end{array}$ & $\begin{array}{c}\text { Qualitativo ou } \\
\text { Quantitativo }\end{array}$ & Incorporado \\
\hline $\begin{array}{c}\text { Transformativa } \\
\text { concomitante }\end{array}$ & $\begin{array}{c}\text { Qualitativo e } \\
\text { Quantitativo }\end{array}$ & $\begin{array}{c}\text { Integrado, } \\
\text { incorporado ou } \\
\text { conectado }\end{array}$ & Explícita & $\begin{array}{c}\text { Coleta de dados qualitativos e quantitativos de modo } \\
\text { Os dados qualitativos e quantitativos são coletados de modo } \\
\text { concomitante e é guiado por uma perspectiva teórica explícita. }\end{array}$ \\
\hline
\end{tabular}

Fonte: Creswell (2010). 
Filosófica e metodologicamente, o método sob exame se embasa no pragmatismo, o qual admite quase todos os estudos que fundamentam as pesquisas quantitativas e qualitativas. De acordo com Greene (2007), o ‘coração’ do pragmatismo (e, portanto, da visão mista) é convidar vários 'modelos mentais' para ocuparem o mesmo espaço de busca e estabelecer um diálogo respeitoso em que os enfoques se alimentem mutuamente, além de, coletivamente, produzirem melhor sentido de compreensão do fenômeno estudado. 0 pragmatismo envolve uma multiplicidade de perspectivas, premissas teóricas, tradições metodológicas, técnicas de coleta e análise de indicadores, entendimentos e valores que constituem os elementos dos modelos mentais. Nesse âmbito, Sampieri e Mendoza (2018) acrescentam que, às vezes, essas duas aproximações do conhecimento parecem ser contraditórias, mas talvez o que se vê como sendo contraditório seja simplesmente uma complementação.

Por fim, mostra-se salutar aduzir a essas discussões alguns dos desafios inerentes ao desenvolvimento de um estudo misto. Creswell e Clark (2013) ressaltam que o desenvolvimento desse tipo de experimento demanda do pesquisador habilidades com métodos qualitativos e quantitativos, tanto para coleta e análise como a fim de validar os dados. Outro aspecto a merecer oportuna ênfase é o fato de que demandas mistas exigem mais tempo, recursos e, consequentemente, esforços extensivos. Além disso, o caráter recente da metodologia citada, na realidade contemporânea, reclama a necessidade de mostrar o valor desse modelo de proceder à busca.

\section{Percurso metodológico}

Este ensaio caracteriza-se como documental, haja vista que esse tipo de investigação possibilita se “[...] compreender uma dada realidade não em sua concretização imediata, mas de forma indireta, por meio da análise de documentos produzidos pelo homem a seu respeito" (MENDES; FARIAS; NÓBREGA-THERRIEN, 2011, p. 32). No método da pesquisa documental, pode-se recorrer à análise de distintos documentos impressos ou digitais, como livros, revistas, textos acadêmicos, diários, anotações, fotografias, pinturas, entre outros. Segundo McCulloch (2011, p. 249), "um documento pode ser definido resumidamente como um registro de um evento ou processo"7.

No texto sob relato, os documentos de interesse restringiram-se às teses de um programa de pós-graduação específico, publicadas nos últimos seis anos (2013-2018). As buscas foram realizadas no sítio da Plataforma Sucupira e, conforme disposto na Tabela 1, foram identificadas, ao todo, 104 teses produzidas no âmbito do PPGE analisado. Identificou-se o fato de que, destas, 11 (10,6\%) recorrem à abordagem mista, submetendose, portanto, à análise neste estudo.

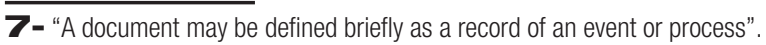


Tabela 1 - Síntese das buscas realizadas

\begin{tabular}{|c|c|c|c|}
\hline PERÍODO/ANO & TESES PRODUZIDAS & TESES COM ABORDAGEM MISTA & $\%$ \\
\hline 2013 & 20 & 03 & 15,0 \\
\hline 2014 & 17 & - & - \\
\hline 2015 & 22 & 04 & 18,2 \\
\hline 2016 & 14 & 02 & 14,3 \\
\hline 2017 & 15 & 02 & 13,3 \\
\hline 2018 & 16 & - & - \\
\hline TOTAL & 104 & 11 & 10,6 \\
\hline
\end{tabular}

Fonte: https://sucupira.capes.gov.br/sucupira/

Finalizadas as buscas, os passos seguintes consistiram em uma imersão na bibliografia alusiva ao método misto, mediante visitação às obras de Creswell (2010), Creswell e Clark (2013), Sampieri, Collado e Lúcio (2013), Sampieri e Mendoza (2018), dentre outros, sequenciando-se o exame das teses selecionadas. Recorreu-se à literatura especializada para o estabelecimento de critérios de análise das referidas teses e optouse pelas orientações de Creswell (2012), haja vista que esse autor propõe a definição e a descrição básica dessa abordagem, além de enumerar uma lista de questionamentos que devem orientar o pesquisador durante o planejamento de um procedimento desta natureza (Quadro 3).

Quadro 3 - Lista de questões para o planejamento de um procedimento de métodos mistos

\begin{tabular}{l:l}
\hline 1 & Apresentou-se uma definição básica da pesquisa de métodos mistos? \\
\hline 2 & Apresentou-se uma razão para o uso concomitante das abordagens (ou dados) quantitativa e qualitativa? \\
\hline 3 & O leitor tem uma percepção do uso potencial de um projeto de métodos mistos? \\
\hline 4 & São identificados os critérios para a escolha de uma estratégia de métodos mistos? \\
\hline 5 & A estratégia é identificada e foram mencionados os critérios para escolha? \\
\hline 6 & Apresentou-se um modelo visual que esclareça a estratégia de pesquisa? \\
\hline 7 & Utilizou-se a notação apropriada na apresentação do modelo visual? \\
\hline 8 & São mencionados os procedimentos de coleta e análise dos dados da forma como eles se relacionam com o modelo? \\
\hline 9 & São mencionadas as estratégias de amostragem para a coleta de dados quantitativos e qualitativos? Eles estão relacionados à estratégia? \\
\hline 10 & São discutidos os procedimentos para validação dos dados quantitativos e qualitativos? \\
\hline 11 & É mencionada a estrutura narrativa, e ela está relacionada ao tipo de estratégia de métodos mistos que está sendo utilizada?
\end{tabular}

Fonte: Creswell (2012, p. 240).

No quadro acima, Creswell (2012) exibe uma lista de indagações para o planejamento de um estudo de métodos mistos, preconizando o avanço da natureza desta configuração, o tipo de estratégia que está sendo proposto para o estudo, os procedimentos específicos para coleta, análise, validação dos dados e modos de seleção da amostragem, além de incluir a necessidade de um modelo visual dessa abordagem, o papel do pesquisador e a estrutura do relatório final. Optou-se, portanto, por analisar as teses selecionadas com base nesses pressupostos, de modo que a próxima seção está estruturada no intuito de 
identificar as preocupações metodológicas dos autores das teses sob análise para com os fundamentos do método adotado em suas investigações.

Os questionamentos de Creswell, dispostos no Quadro 1, anteriormente mencionado, foram, portanto, a bússola para a análise das teses selecionadas. Os indicativos referentes a cada um desses questionamentos são apontados na seção seguinte, de modo quantitativo e qualitativo, agrupados em subseções, por semelhança e/ou complementaridade. Em cada subseção, foram dispostas as análises das teses selecionadas, no intuito de identificar como se enquadram nas orientações de Creswell (2012) para o planejamento e formulação de uma pesquisa de métodos mistos, orientando o leitor na compreensão dos principais pressupostos de elaboração de um estudo de tal natureza, no PPGE analisado, assim como os aspectos negligenciados/secundarizados.

Como fechamento dessas reflexões preliminares, destaca-se que, com vistas a adequarse aos preceitos éticos, as teses selecionadas foram nominadas sob as terminologias A, B, C, ..., pois, de acordo com Creswell (2012), os pesquisadores precisam respeitar os locais de pesquisa, deixando-os intactos após um estudo, havendo a necessidade de prever a possibilidade de que informações prejudiciais sejam reveladas durante o recolhimento de dados.

\section{O diálogo com/sobre as teses selecionadas}

\section{Definição básica da pesquisa de métodos mistos e razão para o uso concomitante das abordagens qualitativa e quantitativa}

Conforme disposto no Gráfico 1, abaixo, cinco teses analisadas $(45,5 \%)$ não contêm uma defınição básica de pesquisa de métodos mistos (A, B, C, F e G). As outras seis (54,5\%) encerram uma conceituação para esse tipo de estudo, recorrendo aos seguintes autores: Minayo (2010) na tese D; Minayo, Deslandes e Gomes (2007) na tese E; Minayo e Sanches (1993) na tese H; Minayo (1994) na tese I; Minayo, Deslandes e Gomes (2007) na tese J e Creswell (2012) na tese K.

Gráfico 1 - Definição de método misto e explicitação para o uso concomitante quali-quanti

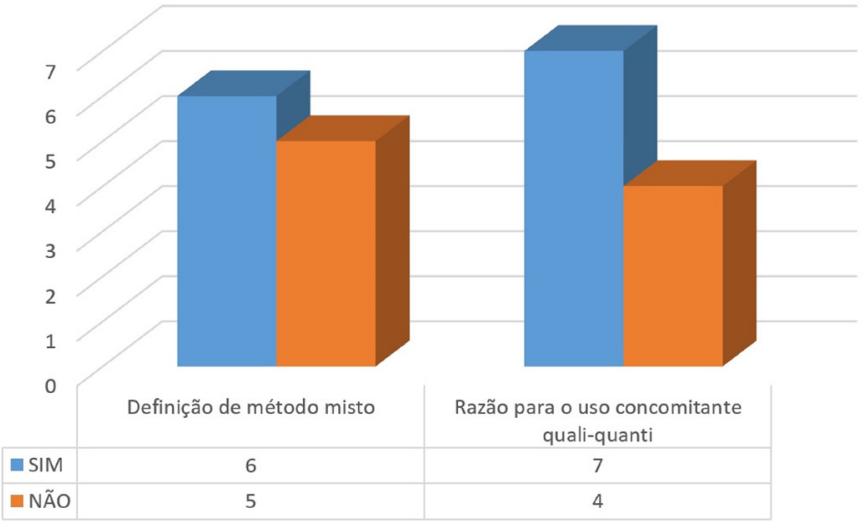

Fonte: Elaboração própria, 2020. 
Observa-se que, embora Sampieri, Collado e Lucio (2013, p. 550) destaquem a existência de uma diversidade de classificações na literatura, tais como "pesquisa qualiquanti, integrativa, multimétodos, estudos de triangulação, métodos múltiplos ou pesquisa mista”, a nomenclatura 'qualitativa-quantitativa' foi a mais recorrente entre as teses analisadas, adotada por oito delas (72,7\%). Apenas uma $(9,1 \%)$ foi caracterizada como mista e duas $(18,2 \%)$ como 'predominantemente qualitativas', mesmo demonstrando todas as características de um estudo do tipo misto, tendo inclusive no decorrer de seu capítulo metodológico caracterizações relacionadas ao âmbito quantitativo da investigação.

Outra informação disposta no Gráfico 1 é a de que a maior parte das teses analisadas (sete; 63,6\%) denota justificativas para o uso concomitante das abordagens qualitativa e quantitativa, dentre as quais é sobrelevado o caráter complementar entre as duas abordagens (ver Quadro 4). Essa perspectiva coaduna-se com a compreensão de Sampieri e Mendonza (2008), quando esses autores evidenciam que os métodos mistos conformam o "terceiro caminho", indo, também, ao encontro do entendimento de Creswell (2012), ao destacar o fato de as abordagens qualitativa e quantitativa possuírem maneiras diferentes de cuidar do mesmo fenômeno, mas, combinadas, minimizam ou até mesmo neutralizam algumas das desvantagens de certas trilhas metodológicas.

Quadro 4 - Justificativas para a utilização concomitante das abordagens quali-quanti

\begin{tabular}{c|ccc}
\hline TESE & JUSTIFICATIVA & AUTORES DE REFERÊNCIA \\
\hline A & Não apresentam & \\
\hline B & & & \\
\hline C & A diferença entre as abordagens é de natureza e não de escala hierárquica, e não são \\
incompatíveis & Minayo (2010) \\
\hline D & Oposição complementar & Minayo (2007) \\
\hline E & Não apresenta & \\
\hline F & A parte quantitativa irá enriquecer a investigação & Não apresenta \\
\hline G & Os âmbitos qualitativos e quantitativos não se excluem & Minayo (1993) \\
\hline H & Não há quantificação sem qualificação & Bauer; Gaskell (2002) \\
\hline I & Complementaridade entre as abordagens qualitativa e quantitativa & Triviños (2010) \\
\hline J & Flick (2004)
\end{tabular}

Fonte: Elaboração própria, 2020.

\section{Identificação dos procedimentos de coleta e análise de dados}

Verificou-se que apenas a tese H não envolve os procedimentos de análise de dados. Nas demais (90,9\%), há tanto a identificação dos procedimentos de coleta como de análise, conforme dispõe o Quadro 5. 
Quadro 5 - Procedimentos de coleta e análise de dados utilizados nas teses investigadas

\begin{tabular}{|c|c|c|c|}
\hline TESES & CARACTERIZAÇÃO & COLETA DE DADOS & ANÁLISE DE DADOS \\
\hline A & NÃO APRESENTA & $\begin{array}{l}\text { Métodos estatísticos multivariados (ESCOFIER; } \\
\text { PAGÉS, 1992) }\end{array}$ & $\begin{array}{c}\text { Análise de correspondência com o software } \\
\text { Statistical Package for the Social Science } \\
\text { (SPSS) }\end{array}$ \\
\hline B & Conclusiva e etnográfica & $\begin{array}{c}\text { Entrevista estruturada, entrevista } \\
\text { semiestruturada em profundidade; análise } \\
\text { documental }\end{array}$ & $\begin{array}{l}\text { Análise de conteúdo } \\
\text { Análise estatística }\end{array}$ \\
\hline C & Descritiva e documental & $\begin{array}{l}\text { Microdados das instituições analisadas e } \\
\text { documentos em nível nacional e internacional }\end{array}$ & $\begin{array}{c}\text { Análise estatística com uso do LibreBroffice e } \\
\text { número-índice de base } 100\end{array}$ \\
\hline D & Exploratória e estudo de caso & Análise documental; questionário aberto & $\begin{array}{l}\text { Análise de conteúdo; } \\
\text { Escala Likert }\end{array}$ \\
\hline $\mathrm{E}$ & Exploratória e estudo de caso & $\begin{array}{l}\text { Questionário misto, roda de conversa, } \\
\text { entrevista semiestruturada, análise documental, } \\
\text { observação sistemática }\end{array}$ & $\begin{array}{l}\text { Análise textual discursiva com a utilização do } \\
\text { Design Research }\end{array}$ \\
\hline $\mathrm{F}$ & Bibliográfica e documental & Análise documental & $\begin{array}{l}\text { Análise de conteúdo } \\
\text { Análise estatística }\end{array}$ \\
\hline G & $\begin{array}{l}\text { Exploratória e } \\
\text { estudo de caso do tipo } \\
\text { Múltiplo }\end{array}$ & $\begin{array}{c}\text { Entrevista em profundidade, levantamento } \\
\text { de índices da instituição analisada (dados } \\
\text { numéricos) e análise documental }\end{array}$ & $\begin{array}{l}\text { Análise de conteúdo } \\
\text { Análise estatística }\end{array}$ \\
\hline H & $\begin{array}{l}\text { Exploratória, bibliográfica e } \\
\text { documental }\end{array}$ & Análise de documentos & $\begin{array}{c}\text { Não cita os procedimentos de análise } \\
\text { qualitativos nem quantitativos, mas apresenta } \\
\text { muitos dados em percentuais. }\end{array}$ \\
\hline I & NÃO APRESENTA & $\begin{array}{l}\text { Questionário eletrônico (Google Drive); } \\
\text { Memorial }\end{array}$ & $\begin{array}{c}\text { Escala de Likert } \\
\text { Análise de conteúdo }\end{array}$ \\
\hline $\mathrm{J}$ & Exploratória e cartográfica & $\begin{array}{l}\text { Questionário (Google Drive); observação } \\
\text { participante; entrevista aberta; diário de campo }\end{array}$ & $\begin{array}{c}\text { Software NVivo } \\
\text { Queries } \\
\text { Gráficos }\end{array}$ \\
\hline K & NÃO APRESENTA & Entrevista em profundidade; análise documental & $\begin{array}{c}\text { Análise estatística de regressão múltipla; } \\
\text { análise de variância (ANOVA); teste Tukey; } \\
\text { análise proposicional do discurso (análise de } \\
\text { conteúdo) }\end{array}$ \\
\hline
\end{tabular}

Fonte: Elaboração própria, 2020.

Os instrumentos de coleta de indicadores expressam similaridades, visto que, em sua maioria, são utilizados questionários, entrevistas e análise documental. Destaca-se o fato de os autores identificarem o enfoque adotado na utilização de cada um desses instrumentos, se qualitativo ou quantitativo - perspectiva que se coaduna com as orientações de Creswell (2010). Como ensina o autor, é importante que o pesquisador identifique e seja específico sobre os tipos de dados - tanto qualitativos quanto quantitativos - que serão coletados durante o estudo proposto.

Identificou-se, ainda, o fato de as teses $\mathrm{A}$, I e $\mathrm{K}$ não exprimirem claramente uma caracterização da pesquisa, quanto à natureza e ao método adotado (ver Quadro 5). As demais são, em sua maioria, de natureza exploratória (cinco; 45,5\%), diversificando-se 
no tocante ao método, havendo estudos do tipo etnográfico, cartográfico, estudo de caso, bibliográfico e documental.

No que concerne à análise de dados, notou-se que a "análise de conteúdo" - e suas variações - foi o procedimento mais repetido nessas teses, aparecendo em seis $(54,5 \%)$ delas (B, D, F, G, I e K), sempre associadas a instrumentos de análise quantitativos, dentre os quais estão inclusos softwares, que auxiliam os pesquisadores no tratamento de um maior volume de dados, tais como o SPSS (utilizado na tese A). Esse é um "pacote estatístico" que permite realizar análises estatísticas e gráficas com uma amplitude de indicativos, tendo como principais funções a preparação e validação de dados; árvores de decisão; modelos de regressão; padrões estatísticos avançados; tabelas; tendências; categorias; análise geoespacial e funções de simulação (SANTOS, 2019).

$\mathrm{Na}$ tese $\mathrm{B}$, foram realizadas análises individuais qualitativas (análise de conteúdo Laurence Bardin) e quantitativas (análises estatísticas), além do emprego de matrizes de correlação que, de acordo com Creswell (2010), dão oportunidade a uma análise dos dados qualitativos e quantitativos de modo combinado. Nessa circunstância específica, para o tratamento dos dados dessa matriz, foram utilizadas análises estatísticas, mas o autor da referida tese não explicita que instrumento foi aplicado para auxiliar nesse tratamento, especificamente. Essa situação se repete nas teses F e G. Outra peculiaridade, já citada, reside na tese $\mathrm{H}$, pois não são mencionados procedimentos de análise dos dados (sejam qualitativos ou quantitativos).

Característica interessante, no que diz respeito aos procedimentos de análise de dados dos textos sob exame, residiu na tese E, a qual se louvou no Design Research, que, de acordo com Manson (2006), conforma uma metodologia de análise consistente em empregar uma teoria, a fim de projetar artefatos e usar técnicas para avaliá-los, resultando, ao final de um ciclo, numa teoria melhorada. Desse modo, com amparo em um design inicial, os pesquisadores coordenam as atividades junto com os participantes, testam, redesenham e implementam intervenções para aprimorar o design. À medida que a pesquisa avança e um novo "ciclo" é iniciado, o design original é aprimorado, bem como o são as ações e intervenções, aperfeiçoando a prática e avançando nos objetivos teóricos. Configura-se, portanto, numa elaboração criativa de tentativa e erro, em que as proposições de soluções são reiteradas e aprimoradas até serem poderosas o suficiente para constituírem uma solução completa para o problema investigado.

\section{Identificação dos critérios de amostragem e procedimentos de validação dos dados qualitativos e quantitativos}

Minayo (2017) ressalta a importância da amostragem, por estar vinculada à credibilidade metodológica de uma investigação. Verificou-se, com efeito, que todas as teses analisadas expressam critérios para a seleção da amostra, conforme disposto no Gráfico 2. 
Gráfico 2 - Definição dos critérios para seleção da amostra e procedimentos de validação dos dados

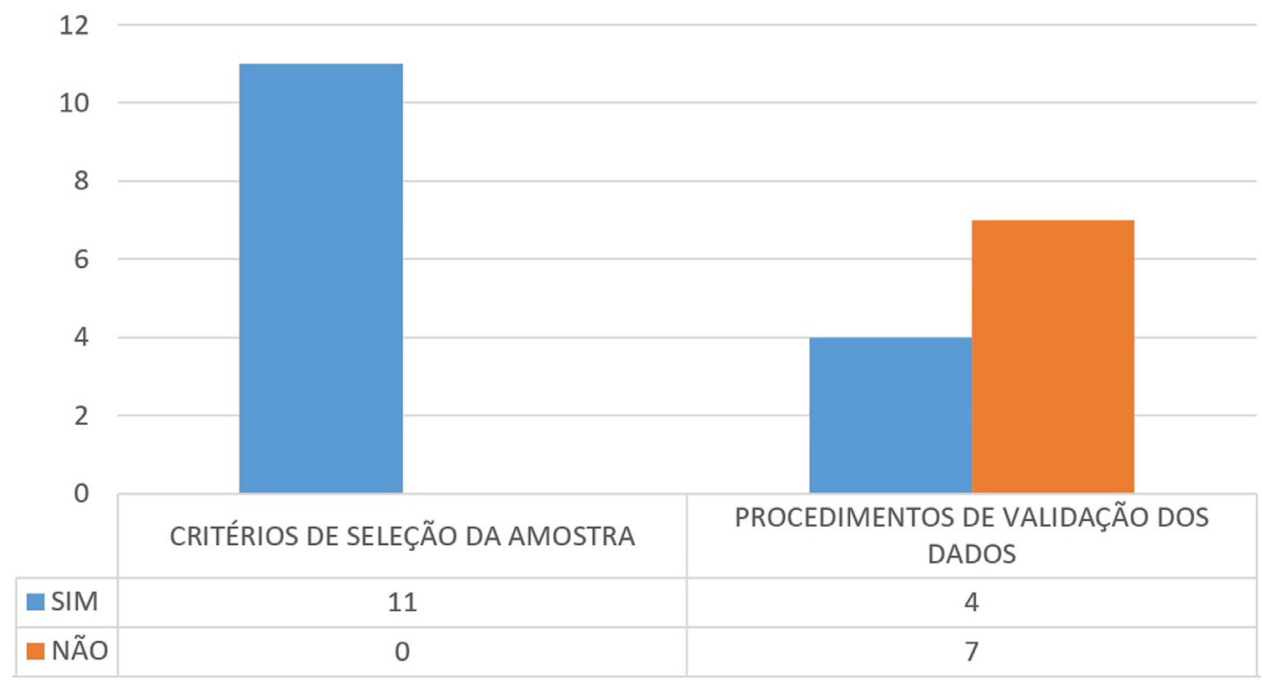

Fonte: Elaboração própria, 2020.

Mostra-se precípuo ressaltar que apenas a tese B determina a classificação do procedimento de amostragem adotado. Nessa situação, aplicam-se dois critérios de seleção da amostra - um para a parte qualitativa da pesquisa e outro de ordem quantitativa. Inicialmente, foi selecionada uma amostra representativa do total de sujeitos pertencentes ao grupo pesquisado (amostra probabilística do tipo estratificada proporcional), enquanto na segunda parte do estudo utilizou-se de sorteios aleatórios para seleção daqueles que comporiam a amostra de coleta de dados qualitativos (amostra probabilística aleatória simples). Essa tese enquadra-se, portanto, nas amostragens probabilísticas.

As outras dez $(90,9 \%)$ teses analisadas não apontam esse detalhamento; mas, mediante sua leitura, foi fácil identificar o fato de que fazem uso de amostragens não probabilísticas, nas quais os pesquisadores se guiaram por critérios previamente estabelecidos, com base nos objetivos do estudo (A, C, D, F, G, H, I e J). Foi dado, ainda, investigar que as teses E e $K$ utilizam uma amostragem não probabilística por conveniência (GIL, 2002), visto que os sujeitos da pesquisa foram selecionados mediante convite e posterior interesse em participar dos estudos.

Quanto aos procedimentos de validação dos dados, conforme disposto no Gráfico 2 , somente quatro $(36,4 \%)$ das teses analisadas (B, G, I e K) fazem menção ou explicitam claramente os procedimentos utilizados para validação dos dados qualitativos e quantitativos. Nas teses B, G e K, os autores se referem à validação de um dos instrumentos de coleta de dados, ressaltando que as entrevistas aplicadas passaram inicialmente por um pré-teste para análise de possíveis adequações a serem inseridas. Em contrapartida, na tese I o instrumento de coleta validado foi um questionário, no qual o pré-teste foi necessário para verificar a análise semântica do enunciado das questões e realizar as modificações necessárias. 
É importante ressaltar que a validação de dados realizada nas teses citadas acima referiu-se somente a um dos métodos (ou qualitativa ou quantitativa), não englobando todas as fases da investigação, conforme orientado na literatura atual. Creswell (2012), assim como outros estudiosos dos métodos mistos, defende o emprego de procedimentos de validação para as fases quantitativa e qualitativa de estudos mistos, de modo que, para os indicadores qualitativos, por exemplo, precisam ser mencionadas as estratégias utilizadas para verificar a precisão dos resultados, o que inclui fontes de dados de triangulação, verificação dos membros, descrição detalhada, dentre outros procedimentos.

\section{Identificação da estratégia de métodos mistos adotada e dos critérios para escolha}

Para Creswell (2012), a adoção de uma estratégia ${ }^{8}$ de métodos mistos demanda uma série de decisões a serem tomadas, em relação: ao enfoque que melhor se adapta à formulação do problema de pesquisa; distribuição do tempo; às maneiras de combinação e peso que os métodos quali e quanti terão no estudo; ao modelo visual que melhor se ajusta; e à estratégia e abordagem que deixam o pesquisador mais confortável para empregar. De tal maneira, deixar claros os critérios considerados pelo pesquisador para a adoção de uma estratégia em detrimento de outra mostra-se essencial para a demonstração do percurso metodológico perfilado em uma investigação mista. Para tanto, neste segmento, foram identificados critérios considerados nas teses em análise, assim como se os autores identificam a(s) estratégia(s) adotada(s) em cada uma delas.

Verificou-se que somente a tese K (9,09\%) identifica a estratégia de pesquisa e os critérios adotados para sua escolha. No trabalho stricto sensu agora mencionado, para identificar a estratégia utilizada em sua investigação, o autor recorre a Creswell (2012, p. 250), classificando-a como de triangulação concomitante, e mostrando sua definição: “[...] o pesquisador coleta concomitantemente os dados qualitativos e quantitativos e depois compara os bancos de dados para determinar se há convergência, diferenças ou alguma combinação [...]". Nesse caso, os dados qualitativos e quantitativos coletados foram comparados à bases de dados educacionais em âmbito nacional.

A justificativa utilizada para a adoção de tal estratégia fundamentou-se nos objetivos da pesquisa, que, conforme destacado pelo autor, necessitam dos dois enfoques (quali e quanti) para serem respondidos, além da necessidade de triangulação dos dados para favorecer uma compreensão mais ampla do fenômeno investigado. Essas perspectivas vão ao encontro dos pressupostos de Creswell e Clark (2013), haja vista que esses autores ressaltam haver problemas de pesquisa mais bem respondidos, usando-se métodos mistos, e destacam a necessidade de que o pesquisador tenha sensibilidade para detectar esse fato.

\section{Apresentação de um modelo visual para a estratégia de pesquisa, mediante notação apropriada}

Detectou-se o fato de que nenhuma das teses estudadas neste experimento indicou uma notação ou explicitou claramente o modelo visual adotado. Como critérios de

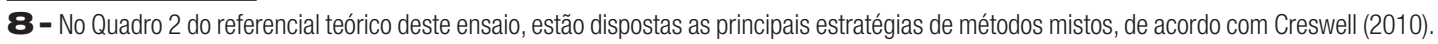


ilustração, todavia, se optou por elaborar, para defender neste escrito, um modelo visual (Figura 1) para o percurso metodológico seguido na única tese na qual o autor identificou a estratégia de pesquisa perfilada em sua investigação: a tese $\mathrm{K}$, conforme explicitado na subseção anterior.

Figura 1 - Modelo visual, com notação, adotado na tese K - projeto de triangulação concomitante

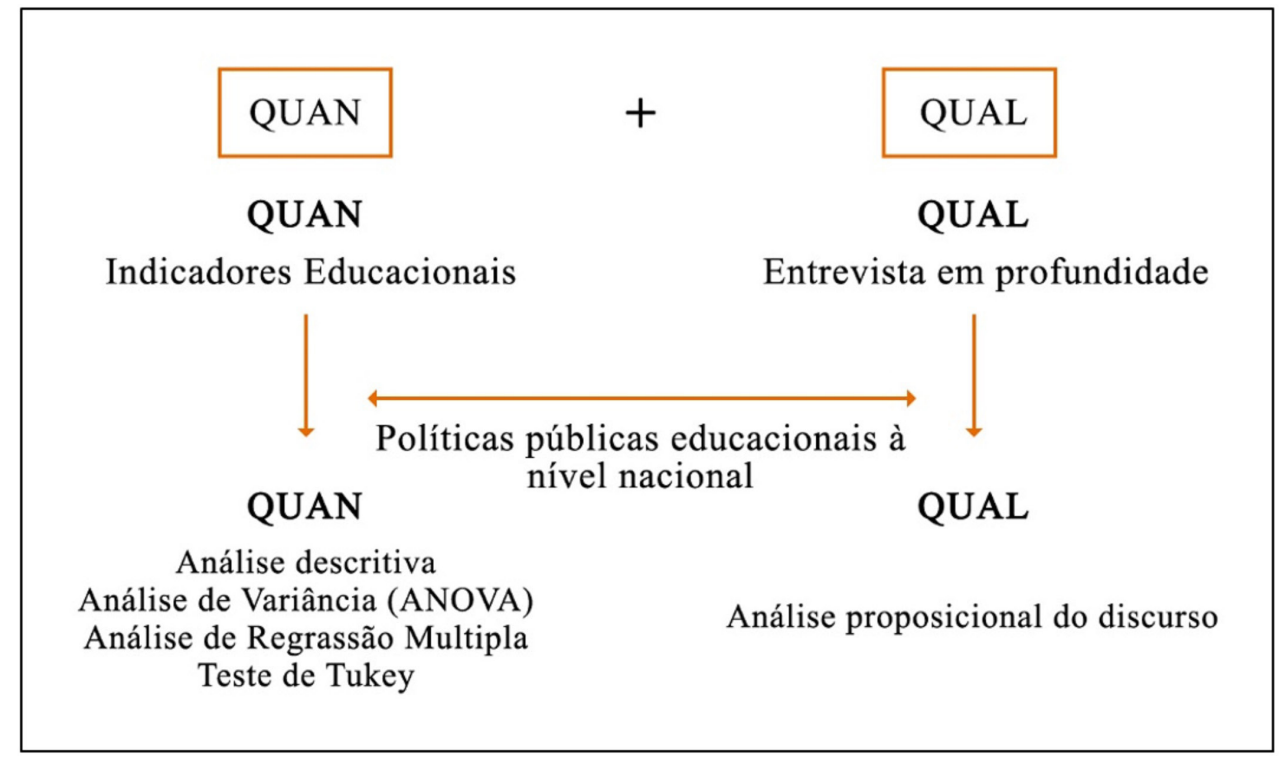

Fonte: Elaboração própria, com suporte em Creswell (2012) e no percurso metodológico da tese K.

No referido experimento, a triangulação concomitante foi a estratégia de pesquisa escolhida, baseando-se no percurso seguido pelo pesquisador e nos escritos de Creswell (2012). 0 modelo visual, anteriormente expresso, ilustra a tese referida, visto que nesse estudo os dados qualitativos e quantitativos assumem peso igual (QUAL/QUAN), são coletados e analisados ao mesmo tempo, mediante mensuração de indicadores educacionais (QUAN) e entrevistas em profundidade (QUAL), para, em seguida, serem analisados e comparados entre si e a bancos de dados educacionais na contextura nacional.

\section{Considerações finais}

A orientação paradigmática para as pesquisas de métodos mistos é algo recente. Além disso, mostra-se desafiador aos pesquisadores tratar em um mesmo trabalho os métodos qualitativo e quantitativo. Mesmo assim, é crescente a produção científica dessa abordagem de pesquisa, de modo que sua popularização é uma realidade em todas as áreas do conhecimento.

Reitera-se, entretanto, com base nas análises realizadas em teses de um programa de pós-graduação em educação, nota sete, de acordo com avaliação da CAPES que, no âmbito do referido programa, emerge a necessidade de maior aproximação teórica e 
metodológica dos doutorandos de uma literatura mais atual no tocante à pesquisa de métodos mistos, pois é usual que, em decorrência do seu caráter recente e do aumento vertiginoso, a modificação/evolução desta metodologia de pesquisa sucede de modo muito rápido. Cabe, portanto, aos pós-graduandos que têm interesse em desenvolver esse tipo de estudo, realizar uma imersão na literatura atual e, sobretudo, considerar a contribuição de experimentos em âmbito internacional, pois é sabido que estudiosos como John W. Creswell, R. Burke Johnson, Anthony J. Onwuegbuzie, Roberto Hernández Sampieri, dentre outros, são referências nesse tipo de pesquisa.

Verifıcou-se, ab initio, que a pesquisa de métodos mistos é pouco utilizada no âmbito do PPGE em foco, uma vez que, entre 104 teses produzidas no período em análise (2013 a 2018), apenas $11(10,6 \%)$ se utilizam do método misto. Ademais, somente uma recorreu à nomenclatura mista, oito (72,7\%) delas caracterizaram-se como qualitativas-quantitativas e duas foram classificadas pelos autores como 'predominantemente qualitativas', mesmo demonstrando todas as características de um estudo do tipo misto, contendo, inclusive, no decorrer de seu capítulo metodológico, caracterizações relacionadas ao âmbito quantitativo da investigação. Ademais, cinco (45,5\%) delas não apontaram uma defınição de pesquisa de métodos mistos e sete $(63,6 \%)$ justificaram a utilização concomitante dos métodos qualitativos e quantitativos, destacando o caráter de complementaridade (ou não oposição) entre esses dois métodos.

A maior parte das oito teses que exprimiram claramente uma caracterização da pesquisa é de natureza exploratória (cinco) e há utilização de métodos de pesquisa diversos - como etnográfico, cartográfico, bibliográfico, documental e estudo de caso. Com exceção da tese $\mathrm{H}$, as demais (dez; 90,9\%) indicaram os instrumentos de coleta e análise de dados, ressaltando aqueles que estariam relacionados às partes qualitativa e quantitativa do trabalho. Os instrumentos de coleta mais utilizados foram questionário, entrevista e análise documental. Para analisar os dados, a "análise de conteúdo" - com suas variações - exerceu maior atuação, sendo adotada em seis (54,5\%) das teses analisadas, ao que se seguiu o emprego de procedimentos de análise quantitativos, tais como softwares para dados quantitativos, Escala Likert, dentre outros. Destaca-se, entretanto, o fato de três das teses analisadas (B, F e G) fazerem menção ao uso de análises estatísticas, mas sem especificar o instrumento utilizado ou a maneira como essas análises foram desenvolvidas.

Todas as teses exprimiram os aspectos considerados na seleção da amostra, mas apenas a tese B explicitou o procedimento de amostragem, classificando-a como probabilística. Nas demais teses, predominou o tipo de amostragem não probabilística. Sobre a validação dos dados, apenas quatro $(36,4 \%)$ teses fizeram menção explícita a esse aspecto. E mesmo estas não se adequaram totalmente às orientações de Creswell (2012), pois esse autor orienta que devem ser explicitados procedimentos de validação de todos os dados (qualitativos e quantitativos) e nas referidas teses a validação esteve relacionada somente a um dos métodos (seja qualitativo ou quantitativo).

Apenas uma das teses expressou a estratégia de pesquisa com métodos mistos adotada e os critérios para escolha, enquanto nenhuma delas indicou um modelo visual para a estratégia tomada ou uma notação adequada. Expressos, os resultados ratificam a necessidade de os pesquisadores do referido programa buscarem referências mais atuais no 
tocante aos estudos do tipo misto, pois diversos pesquisadores, a exemplo de Tashakkori e Teddlie (1998), Sampieri, Collado e Lúcio (2013), Johnson e Onwuebuzie (2004), comungam da compreensão e orientações de Creswell (autor-base a quem se reportou na análise das teses) acerca dos pressupostos que devem amparar o estudo misto.

Outra característica a ser ressaltada repousa no fato de se perceber uma evolução teórica e metodológica, quando se compara o percurso metodológico enveredado pelas teses que se utilizam do método misto no decorrer dessas seis décadas (2013-2018), no referido PPGE, haja vista que, em teses mais atuais, observam-se tanto as menções à literatura internacional, como um maior entrelaçamento entre os âmbitos qualitativo e quantitativo, além de justificativas mais bem fundamentadas para essa utilização.

A modo de fechamento, ressalta-se que, nas teses estudadas, há a possibilidade de o consulente-leitor ter uma percepção do uso potencial de um projeto de métodos mistos (CRESWELL, 2012), em maior e menor grau, de modo que, conforme destacado anteriormente, há uma evolução temporal na melhoria dessa percepção. As teses mais atuais aportam perspectivas e justificativas mais claras para o modo como os métodos qualitativo e quantitativo foram sendo utilizados no decorrer da investigação.

Evidencia-se, ainda, a relevância deste levantamento para a realidade da pósgraduação em educação brasileira e para o âmbito da pesquisa de métodos mistos, destacando-se a importância do rigor metodológico em qualquer abordagem investigativa que se vá adotar, visto esse ser um dos instrumentos de sustentação da cientificidade de uma investigação. Além disso, ao identificar lacunas inerentes à utilização do método misto nas teses em análise, almeja-se orientar/auxiliar os pesquisadores na utilização desta abordagem de pesquisa e, de modo concomitante, agregar contributos para a disseminação das pesquisas de métodos mistos.

\section{Referências}

BAUER, Martin W.; GASKELL, George. Pesquisa qualitativa com texto, imagem e som: um manual prático. Petrópolis: Vozes, 2002.

CRESWELL, John W. Educational research: planning, conducting, and evaluating quantitative and qualitative research. 4. ed. Boston: Pearson, 2012.

CRESWELL, John W. Projeto de pesquisa: método qualitativo, quantitativo e misto. 3. ed. Porto Alegre: Artmed, 2010.

CRESWELL, John W. Qualitative inquiry \& research design: choosing among five approaches. 3. ed. Thousand Oaks: Sage, 2013.

CRESWELL, John W.; CLARK, Vick L. Plano. Pesquisa de métodos mistos. 2. ed. Porto Alegre: Penso, 2013.

CRESWELL, John W. et al. Advanced mixed methods research designs. In: TASHAKKORI, Abbas M.; TEDDLIE, Charles B. (ed.). Handbook of mixed methods in social and behavioral research. Thousand Oaks: Sage, 2003. p. 209-240. 
FLICK, Uwe. Uma introdução à pesquisa qualitativa. 2. ed. Porto Alegre: Bookman, 2004.

GALVÃO, Maria Cristiane Barbosa; PLUYE, Pierre; RICARTE, Ivan Luiz Marques. Métodos de pesquisa mistos e revisões de literatura mistas: conceitos, construção e critérios de avaliação. InCID, Ribeirão Preto, v. 8, n. 2, p. 4-24, set. 2017/fev. 2018. Disponível em: https://www.revistas.usp.br/incid/article/ view/121879/133611. Acesso em: 10 jun. 2019.

GIL, Antonio Carlos. Como elaborar projetos de Pesquisa. 4 ed. São Paulo: Atlas, 2002.

GREENE, Jennifer C. Mixed methods in social inquiry. San Francisco: Jossey-Bass, 2007.

IVANKOVA, Natalyia V. Mixed methods applications in action research: from methods to community action. Thousand Oaks: Sage, 2015.

JOHNSON, R. Burke; ONWUEGBUZIE, Anthony J. Mixed methods research: a research paradigm whose time has come. Educational Researcher, Washington, DC, v. 33, n. 7, p. 14-26, 2004. Disponível em: https://journals.sagepub.com/doi/10.3102/0013189X033007014. Acesso em: 23 fev. 2019.

MANSON, Neil J. Is operations research really research? Orion, v. 22, n. 2, p. 155-180, 2006. Disponível em: https://orion.journals.ac.za/pub/article/view/40. Acesso em: 20 dez. 2019.

MCCULLOCH, Gary. Historical and documentary research in education. In: COHEN, Louis; MANION, Lawrence; MORRISON, Keith. Research methods in education. 7. ed. London: Routledge, 2011. p. 248255.

MENDES, Emanoela Therezinha Bessa; FARIAS, Isabel Maria Sabino de; NÓBREGA-THERRIEN, Silvia Maria. Trabalhando com materiais diversos e exercitando o domínio da leitura: a pesquisa bibliográfica e a pesquisa documental. In: NÓBREGA-THERRIEN, Silvia Maria; FARIAS, Isabel Maria Sabino de; NUNES, João Batista Carvalho. Pesquisa científica para iniciantes: caminhando no labirinto. v. 3. Fortaleza: UECE, 2011. p. 25-42.

MINAYO, Maria Cecília de Souza. Amostragem e saturação em pesquisa qualitativa: consensos e controvérsias. Revista Pesquisa Qualitativa, São Paulo, v. 5, n. 7, p. 1-12, abr. 2017. Disponível em: https://edisciplinas.usp.br/pluginfile.php/4111455/mod_resource/content/1/Minayosaturacao.pdf. Acesso em: 20 jun. 2020.

MINAYO, Maria Cecilia de Souza. Ciência, técnica e arte: 0 desafio da pesquisa social. In: MINAYO, Maria Cecilia de Souza (org.). Pesquisa social: teoria, método e criatividade. Petrópolis: Vozes, 1994. p. 9-29.

MINAYO, Maria Cecília de Souza. 0 desafio do conhecimento. São Paulo: Hucitec, 2010.

MINAYO, Maria Cecília de Souza; DESLANDES, Suely Ferreira; GOMES, Romeu. Pesquisa social: teoria, método e criatividade. 26. ed. Petrópolis: Vozes, 2007.

MINAYO, Maria Cecília de Souza; DESLANDES, Suely Ferreira; GOMES, Romeu. Pesquisa social: teoria, método e criatividade. 28. ed. Petrópolis: Vozes, 2009. 
MINAYO, Maria Cecilia de Souza; SANCHES, Odécio. Quantitativo-qualitativo: oposição ou complementaridade? Cadernos de Saúde Pública, Rio de Janeiro, v. 9, n. 3, p. 237-248, set. 1993. Disponível em: http://www.scielo.br/scielo.php?script=sci_a rttext\&pid=S0102-311X1993000300002\&l ng=en\&nrm=iso. Acesso em: 20 abr. 2021.

SAMPIERI, Roberto Hernández; COLLADO, Carlos Fernández; LUCIO, Maria del Pilar Baptista. Metodologia de pesquisa. 5. ed. São Paulo: McGraw-Hill, 2013.

SAMPIERI, Hernández Roberto; MEND0ZA, Paulina Christian. Metodología de la investigación: las rutas cuantitativas, cualitativas y mixta. 1. ed. México, DC: McGraw-Hill, 2018.

SANTOS, Alexandre. IBM SPSS como ferramenta de pesquisa quantitativa. São Paulo: PUC, 2019. Disponível em: https://www.pucsp.br/sites/default/files/download/posgraduacao/programas/ administracao/IBM-SPSS-como-ferramenta\%20de-pesquisa-quantitativa-alexandra-santos.pdf. Acesso em: 10 jul. 2019.

TASHAKKORI, Abbas M.; CRESWELL, John W. The new era of mixed methods. Journal of Mixed Methods Research, Michigan, v. 1, n. 3, p. 4-7, 2007. Disponível em: https://journals.sagepub.com/doi/ pdf/10.1177/2345678906293042. Acesso em: 12 jun. 2020.

TASHAKKORI, Abbas M.; TEDDLIE, Charles B. Mixed methodology: combining qualitative and quantitative approaches. London: Sage, 1998. (Social research methods series; 46).

TRIVIÑOS, Augusto Nibaldo Silva. Introdução à pesquisa e ciências sociais: a pesquisa qualitativa em educação. 1. ed. São Paulo: Atlas, 2010.

YIN, Robert K. Estudo de caso: planejamento e métodos. 3. ed. Porto Alegre: Bookman, 2005.

Recebido em: 20.09.2020

Revisado em: 24.11.2020

Aprovado em: 10.02.2021

Luciana Rodrigues Leite é doutoranda em educação pelo Programa de Pós-graduação em Educação da Universidade Estadual do Ceará (PPGE/UECE). É professora assistente D do curso de química da Universidade Estadual Vale do Acaraú (UVA), Sobral-CE.

Ana Paula dos Santos Reinaldo Verde é doutoranda em educação pelo Programa de Pósgraduação em Educação da Universidade Estadual do Ceará (PPGE/UECE). É professora da educação básica do estado do Maranhão, São Luis-MA.

Francisco das Chagas Rodrigues de Oliveira é doutorando em educação pelo Programa de Pós-graduação em Educação da Universidade Estadual do Ceará (PPGE/UECE). É professor efetivo da Rede Municipal de Boa Viagem e Monsenhor Tabosa, Ceará. 
João Batista Carvalho Nunes é doutor em filosofia e ciências da educação pela Universidade de Santiago de Compostela (Espanha). É professor do Programa de Pós-Graduação em Educação da Universidade Estadual do Ceará (PPGE/UECE), Fortaleza, CE. 